Article

\title{
Design and Evaluation of Double-Stage Energy Harvesting Floor Tile
}

\author{
Don Isarakorn ${ }^{1, *}$, Subhawat Jayasvasti ${ }^{1}$, Phosy Panthongsy ${ }^{1}$, Pattanaphong Janphuang ${ }^{2}$ and \\ Kazuhiko Hamamoto ${ }^{3}$ \\ 1 Department of Instrumentation and Control Engineering, Faculty of Engineering, King Mongkut's Institute \\ of Technology Ladkrabang, Bangkok 10520, Thailand; s.jayasvasti@gmail.com (S.J.); \\ phosy_pts@hotmail.com (P.P.) \\ 2 Synchrotron Light Research Institute, 111 University Avenue, Muang District, Nakhon Ratchasima 30000, \\ Thailand; pattanaphong@slri.or.th \\ 3 Department of Information Media Technology, School of Information and Telecommunication Engineering, \\ Tokai University, Takanawa Minato-ku, Tokyo 108-8619, Japan; hama@keyaki.cc.u-tokai.ac.jp \\ * Correspondence: don.is@kmitl.ac.th
}

Received: 12 August 2019; Accepted: 2 October 2019; Published: 10 October 2019

check for updates

\begin{abstract}
This paper introduces the design and characterization of a double-stage energy harvesting floor tile that uses a piezoelectric cantilever to generate electricity from human footsteps. A frequency up-conversion principle, in the form of an overshooting piezoelectric cantilever, plucked with a proof mass is utilized to increase energy conversion efficiency. The overshoot of the proof mass is implemented by a mechanical impact between a moving cover plate and a stopper to prevent damage to the plucked piezoelectric element. In an experiment, the piezoelectric cantilever of a floor tile prototype was excited by a pneumatic actuator that simulated human footsteps. The key parameters affecting the electrical power and energy outputs were investigated by actuating the prototype with a few kinds of excitation input. It was found that, when actuated by a single simulated footstep, the prototype was able to produce electrical power and energy in two stages. The cantilever resonated at a frequency of $14.08 \mathrm{~Hz}$. The output electricity was directly proportional to the acceleration of the moving cover plate and the gap between the cover plate and the stopper. An average power of $0.82 \mathrm{~mW}$ and a total energy of $2.40 \mathrm{~mJ}$ were obtained at an acceleration of $0.93 \mathrm{~g}$ and a gap of $4 \mathrm{~mm}$. The prototype had a simple structure and was able to operate over a wide range of frequencies.
\end{abstract}

Keywords: frequency up-conversion; piezoelectric energy harvester; double-stage energy harvesting mechanism; low and variable-frequency vibration energy harvesting

\section{Introduction}

With the recent miniaturization and low-power consumption trends in electronic technology, the realization of autonomous energy devices, especially self-powered wireless sensors for the industry, agriculture, and healthcare, has attracted a lot of interest and considerably increased over the last decade. Utilization of an autonomous energy device is possible only by combining a low-power electronic device with a sustainable electrical energy source. Sustainable electrical energy is commonly extracted from several kinds of ambient environment energies (e.g., solar, thermal, wind, and kinetic energies) through a particular transduction mechanism known as energy harvesting (e.g., photovoltaic, thermoelectric, piezoelectric, electromagnetic, electrostatic, and triboelectric energy harvesting) [1-13]. While there are many kinds of energy harvesters for converting different kinds of ambient environment energy, the piezoelectric energy harvester, which converts mechanical vibration energy into electrical energy, has been widely investigated because it can provide high-power density and high energy 
conversion efficiency with its simple structure $[14,15]$. Moreover, sources of vibration energy are ubiquitous and can be easily harvested from an ambient environment.

In general, many piezoelectric energy harvesters must face the challenge of low and variable frequency vibration, especially of human movement, i.e., the frequency of a vibration source usually does not match with the resonant frequency of a harvester, resulting in low energy conversion efficiency. Several researchers have recently attempted to address this problem in several piezoelectric frequency up-converting energy harvesters [16-24], which always oscillated at their resonant frequency no matter what the frequency of the vibration source was. Their basic structure can be divided into two main parts: a frequency up-converting mechanism and a piezoelectric cantilever or disc. Usually, the function of a frequency up-converting mechanism is to induce an initial deflection of a piezoelectric cantilever or disc by a non-contact magnetic interaction or a mechanical contact and then leave the cantilever to freely oscillate at its resonant frequency. Likewise, in one of our previous works, the present authors fabricated an energy harvesting floor tile with a unimorph PZT cantilever to convert kinetic energy from human footsteps into usable electricity [25]. The initial deflection of the piezoelectric cantilever was induced by the interaction between a permanent magnet and an iron bar. As the iron bar was instantly moved away from the permanent magnet at the tip of the piezoelectric cantilever, the cantilever was left to freely oscillate at its resonant frequency of $10.54 \mathrm{~Hz}$. The frequency up-conversion of the harvester was effective, but a limitation was found. The fabricated floor tile was able to generate energy only after the foot moved up and away from it; landing a foot on it just induced the initial deflection of the piezoelectric cantilever. Therefore, the realization of a double-stage energy harvesting floor tile that can generate energy from both actions of a footstep, with an output voltage waveform, as illustrated in Figure 1, was very desirable and challenging. Frequency up-converting energy harvesters that would likely generate energy in double stages have been presented by several authors. For example, Pozzi et al. [26] introduced a rotary piezoelectric energy harvester for harvesting energy from knee-joint motion where the extension and flexion of the knee would make a plastic plectrum pluck piezoelectric bimorphs. Janphuang et al. [27] studied energy harvesting from a rotating gear using piezoelectric microelectromechanical system harvesters. The teeth of a rotating gear driven by an oscillating mass were able to pluck a piezoelectric cantilever in both clockwise and counterclockwise directions. Both energy harvesting devices were based on a contact frequency up-conversion mechanism that could pluck a piezoelectric cantilever effectively. However, this kind of mechanism reduced the longevity of the bimorph. Kuang et al. [28] reported a knee-joint energy harvester using non-contact magnetic interaction instead of mechanical contact to excite bimorphs. The knee-joint motion actuated a primary magnet to pluck the bimorph through a secondary magnet attached to the bimorph tip. It was able to make the bimorph last longer, but the generated energy significantly dropped at a high speed of magnetic plucking.
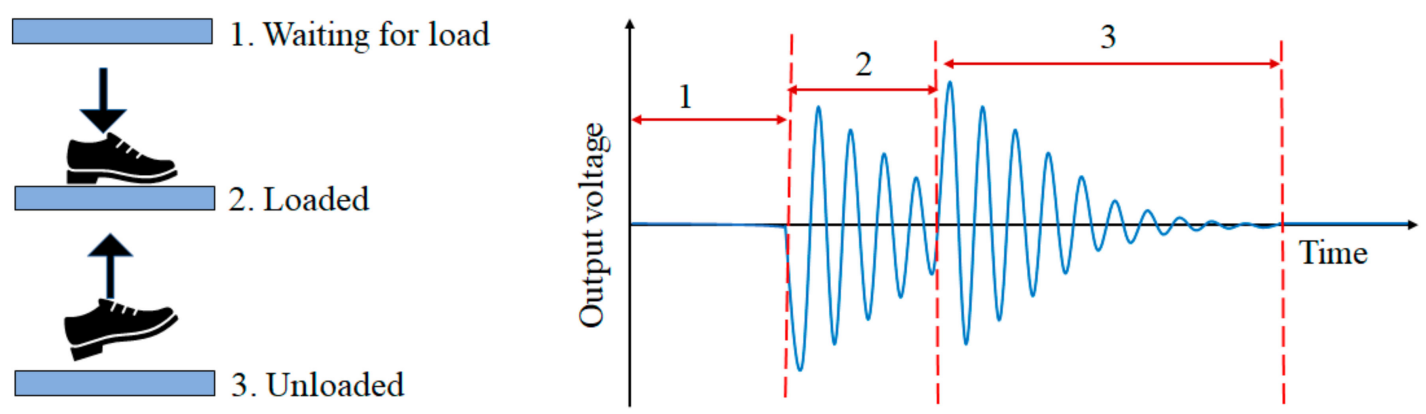

Figure 1. Typical voltage waveforms generated by the double-stage energy harvesting floor tile.

The main aim of this work was to evaluate the feasibility of the designed double-stage energy harvesting floor tile. The overshoot of a proof mass from a mechanical impact between a moving cover plate and a stopper was used to provide piezoelectric frequency up-conversion: the mechanism provides effective plucks on the piezoelectric cantilever that would give it not only a long lifespan 
but also high energy generation. In this paper, the overall mechanism and the operational strategy of the double-stage energy harvesting floor tile are introduced first. Then, the fabricated prototype is depicted, and the experimental methods for investigating the energy harvesting performances are described, followed by the simulated input excitation modes that were based on the behavior of the pedestrians stepping on the floor tile and the configuration of the mechanism. Next, the experimental results are discussed. Lastly, the conclusion is given.

\section{Schematic Description of Double-Stage Energy Harvesting Floor Tile and Its Operation}

Figure 2a illustrates the basic components of the double-stage energy harvesting floor tile. The cover plate is a movable part, which is set to move in the vertical direction-down when stepped on and up when the spring pushes it back. Displacement of the moving-downward cover plate is limited by stoppers. One end of a piezoelectric cantilever is mounted horizontally to a holder mounted underneath the cover plate. The other end is inserted into a proof mass that is used for inducing the initial deflection of the piezoelectric cantilever and increasing mechanical strain in the piezoelectric material as the assembly oscillates. Increased mechanical strain in the piezoelectric material leads to an increase in electrical energy output. The operational strategy is that the piezoelectric cantilever will be induced to oscillate two times when the tile is stepped on only once. When a foot steps down on the tile, a cover plate moves down together with the piezoelectric cantilever and the proof mass and compresses the springs. Immediately, the cover plate is stopped by the stoppers (the walls of the tile box), and the proof mass is pushed down from its equilibrium position and creates a strain on the piezoelectric cantilever in the first deflection of the piezoelectric cantilever, as shown in Figure $2 b$. Next, the piezoelectric cantilever freely oscillates from its restoring force. That is the first oscillation. Illustrated in Figure 2c, when the foot moves up and away from the floor tile, the springs rapidly push the cover plate up to their normal length. As the moving-up cover plate stops as the spring returns to its normal length, the proof mass is pushed up and overshoots its equilibrium position again, but in the opposite direction. Hence, the piezoelectric cantilever is induced to freely oscillate for the second time.

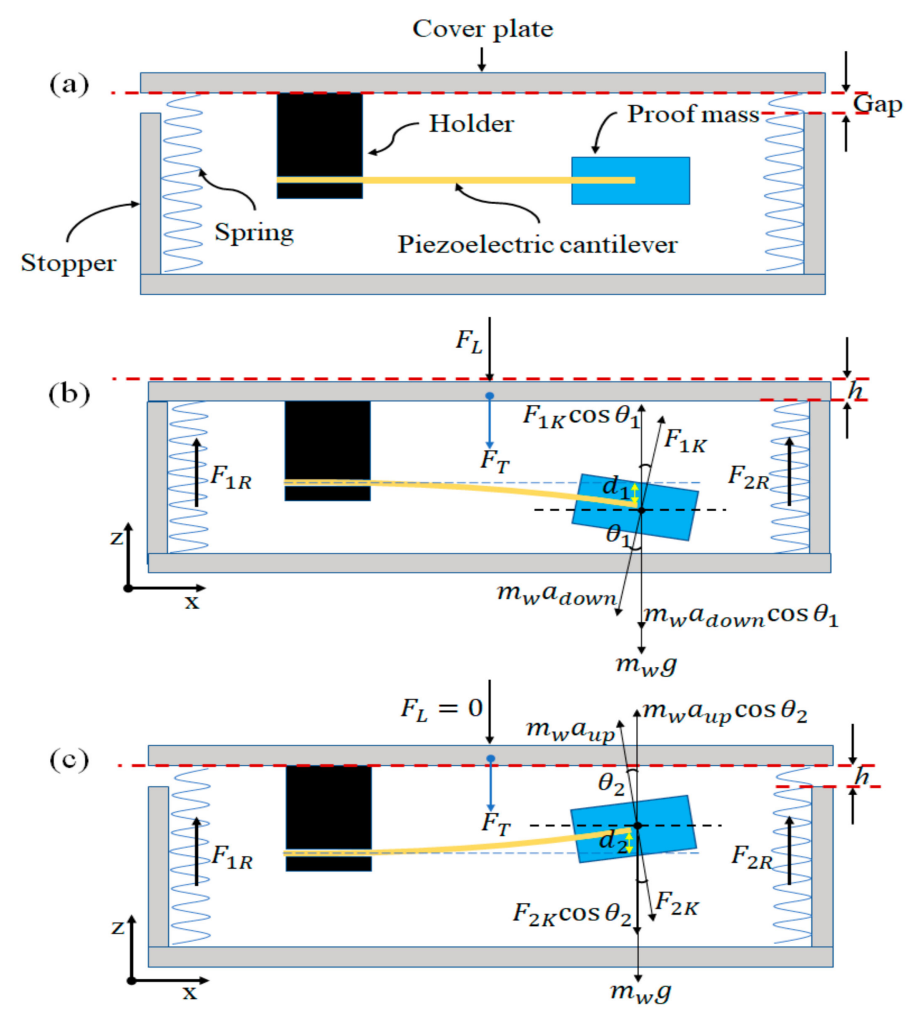

Figure 2. Schematic drawing of the double-stage energy harvesting floor tile: (a) basic components; (b) and (c) are the forces involved in the first and the second deflections of the piezoelectric cantilever. 
In theory, in pushing down the cover plate, the amount of force from a footstep $\left(F_{L}\right)$ compressing the springs from their equilibrium position should be larger than the sum of the restoring forces of the springs $\left(F_{i R}\right), F_{L}>\sum_{i=1}^{n} F_{i R}$. Thus, the necessary force from the cover plate moving down along the $z$-axis $\left(F_{1 Z}\right)$ pressing the springs can be calculated by the following equations of Newton's second law and Hooke's law,

$$
\begin{gathered}
F_{1 Z}=\left(F_{L}+F_{T}\right)-\sum_{i=1}^{n} F_{i R}, \\
F_{1 Z}=\left(m_{L}+m_{T}\right) a_{\text {down }}-\sum_{i=1}^{n} k_{i} h
\end{gathered}
$$

where $F_{T}$ is the damping force exerted by the total mass of the cover plate, piezoelectric cantilever, and the proof mass; $m_{T}$ is the total mass mentioned; subscript $i$ identifies a particular spring; $n$ is the total number of springs used; $k_{i}$, is the spring constant; $h$ is the displacement from the equilibrium position of the spring or the displacement of the moving cover plate; $m_{L}$ is the mass of the person stepping on the tile; and $a_{\text {down }}$ is the downward acceleration of cover plate and its attachment from the tile being stepped on. It can be found by the equation below,

$$
a_{\text {down }}=\frac{F_{1 Z}-\sum_{i=1}^{n} k_{i} h}{m_{L}+m_{T}} .
$$

As the cover plate is stopped by stoppers as shown in Figure $2 b$, the proof mass overshoots its equilibrium position with the downward acceleration $\left(a_{\text {down }}\right)$, deflecting the piezoelectric cantilever for the first time. The force $F_{2 Z}$ along the $z$-axis that induces this first deflection is given by the following equations,

$$
\begin{aligned}
& F_{2 Z}=\left(m_{w} a_{\text {down }} \cos \theta_{1}+m_{w w} g\right)-F_{1 K} \cos \theta_{1}, \\
& F_{2 Z}=m_{w}\left(a_{\text {down }} \cos \theta_{1}+g\right)-k_{\text {eff }} d_{1} \cos \theta_{1},
\end{aligned}
$$

where $m_{w}$ is the weight of the proof mass; $F_{1 K}$ is the restoring force of the piezoelectric cantilever in this deflection [14]; $k_{\text {eff }}$ is the effective spring constant of the piezoelectric cantilever; and $d_{1}$ and $\theta_{1}$ are the tip displacement of the piezoelectric cantilever and the top surface angle of the proof mass in this deflection, respectively. After that, the piezoelectric cantilever freely oscillates by the $F_{1 K}$ force.

After the landing of the step, when the foot moves up and away from the tile, $F_{L}=0$, and the sum of the restoring forces $\left(\sum_{i=1}^{n} F_{i R}\right)$ of the springs is much higher than the damping force $\left(F_{T}\right)$, pushing the cover plate up rapidly. The moving-up force $\left(F_{3 Z}\right)$ of the cover plate along the $z$-axis is calculated by the expression below,

$$
F_{3 Z}=F_{T}-\sum_{i=1}^{n} F_{i R}
$$

The moving-up acceleration $\left(a_{u p}\right)$ can be found as follows,

$$
a_{u p}=\frac{F_{3 Z}}{m_{T}} .
$$

Thus, immediately after the moving-up cover plate stops when the springs return to their normal length, the proof mass overshoots its equilibrium position in the up direction with an acceleration $\left(a_{u p}\right)$, inducing the second deflection of the piezoelectric cantilever as illustrated in Figure 2c. The force $\left(F_{4 Z}\right)$ along the $z$-axis deflecting the piezoelectric cantilever for the second time can be expressed as,

$$
F_{4 Z}=a_{u p} m_{w} \cos \theta_{2}-\left(F_{2 K} \cos \theta_{2}+m_{w} g\right),
$$




$$
F_{4 Z}=m_{w}\left(a_{u p} \cos \theta_{2}-g\right)-k_{e f f} d_{2}
$$

where $F_{2 K}$ is the restoring force of the piezoelectric cantilever in the second deflection [14], while $\theta_{2}$ and $d_{2}$ are the top surface angles of the proof mass and the tip displacement of the piezoelectric cantilever in the second deflection, respectively. Consequently, the piezoelectric cantilever is induced to freely oscillate for the second time by the $F_{2 K}$ force.

\section{Experimental Methods}

To evaluate the feasibility of the double-stage energy harvesting floor tile, the following structure with one piezoelectric cantilever was constructed, as shown in the schematic drawing in Figure 3. A $71 \times 25.4 \times 0.76 \mathrm{~mm}^{3}$ piezoelectric bimorph cantilever (PPA-2011, Midé Technology) was mounted to a holder with a free length of $48 \mathrm{~mm}$ while its free end was attached to a $30 \times 26 \times 6 \mathrm{~mm}^{3}$ proof mass (416 stainless-steel). The main reasons for selecting this commercial bimorph were that it was assembled from two active PZT-5H layers and three FR4 elastic layers with a high-performance aerospace-grade structural epoxy with excellent adhesion, providing robustness, extended durability, and high output power; moreover, it came with mounting holes, simplifying the wiring and mounting to the holder and proof mass [29]. A stainless-steel compression spring with a size of 0.4 (wire diameter) $\times 4$ (out diameter) $\times 25$ (length) $\mathrm{mm}^{3}$ was vertically placed at each corner of the tile. To perform the energy harvesting experiments, a pneumatic actuator was mounted to the prototype to simulate human footsteps, as shown in Figure 4. The pressure of compressed air in a pneumatic actuator, which pushed and accelerated the cover plate down at various speeds, was controlled by a pressure valve regulator, simulating the steps of a pedestrian. The acceleration amplitude of the moving cover plate was measured with an accelerometer (EI-CALC). In addition, the piezoelectric cantilever was connected in series with a load resistor $R_{L}$, as shown in Figure 5, for measuring the output power and energy. The main reason for using a resistive load instead of an impedance load was to simplify the experiments, improving the design. The output transient voltage $V(t)$ across $R_{L}$, which can be used to calculate the output power and energy calculation, was captured by a recording oscilloscope (Tektronix TD 3032B). The average output power $P_{A v g}$ at time $t_{N}$ can be computed by Equation (10) below [27],

$$
P_{A v g}\left(t_{N}\right)=\frac{1}{t_{N}} \int_{0}^{t_{N}} \frac{V_{L}^{2}(t)}{R_{L}} d t,
$$

where the subscript $N$ denotes the number of voltage samples. The generated energy $E\left(t_{N}\right)$ as a function of time was found by the following expression $[21,28]$ :

$$
E\left(t_{N}\right)=\sum_{m=0}^{N} \frac{V_{L}^{2}\left(t_{m}\right)}{R_{L}} \Delta t_{m} \text { for } N>0,
$$

where $V_{L}\left(t_{m}\right)$ is the voltage across the resistive load at time $t_{m} ; m=0,1,2,3 \ldots$ identifies the voltage sample at a particular time, and $\Delta t_{m}$ is the measurement time interval of the voltage samples.

The energy harvesting performances of the prototype were evaluated with simulated steps based on three parameters: the weight of the pedestrian, gap width between the cover plate and the stopper, and varying low footstep frequency. In the experiment, the pneumatic actuator pushed the cover plate down at several values of acceleration $\left(a_{\text {down }}\right)$ with a fixed gap width $(h)$, resembling pedestrians with different weights stepping on the floor tile; the acceleration of the cover plate was proportional to the weight of pedestrian, as expressed in Equation (3) in Section 2. Similarly, the cover plate was also pushed to move down with a fixed acceleration $\left(a_{\text {down }}\right)$ but with varying gap widths $(h)$, representing the cases where a pedestrian steps on floor tiles twice, in which the distance between the cover plate and stoppers were different. Along the same line, the pneumatic actuator was actuated at several frequencies with the same fixed acceleration to push the cover plate down to meet a gap with a fixed 
width $(h)$, representing the cases where the tile was stepped on at different frequencies. The scenario represented by this test was that the floor tile was installed in an area crowded with people, and so it was stepped on by many pedestrians with varying frequencies.

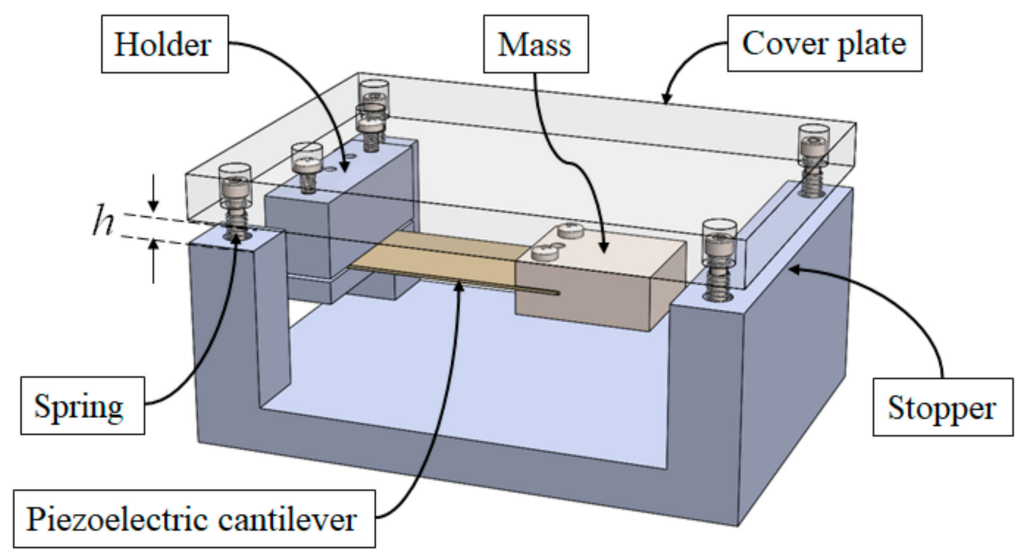

Figure 3. Schematic drawing of the prototype.

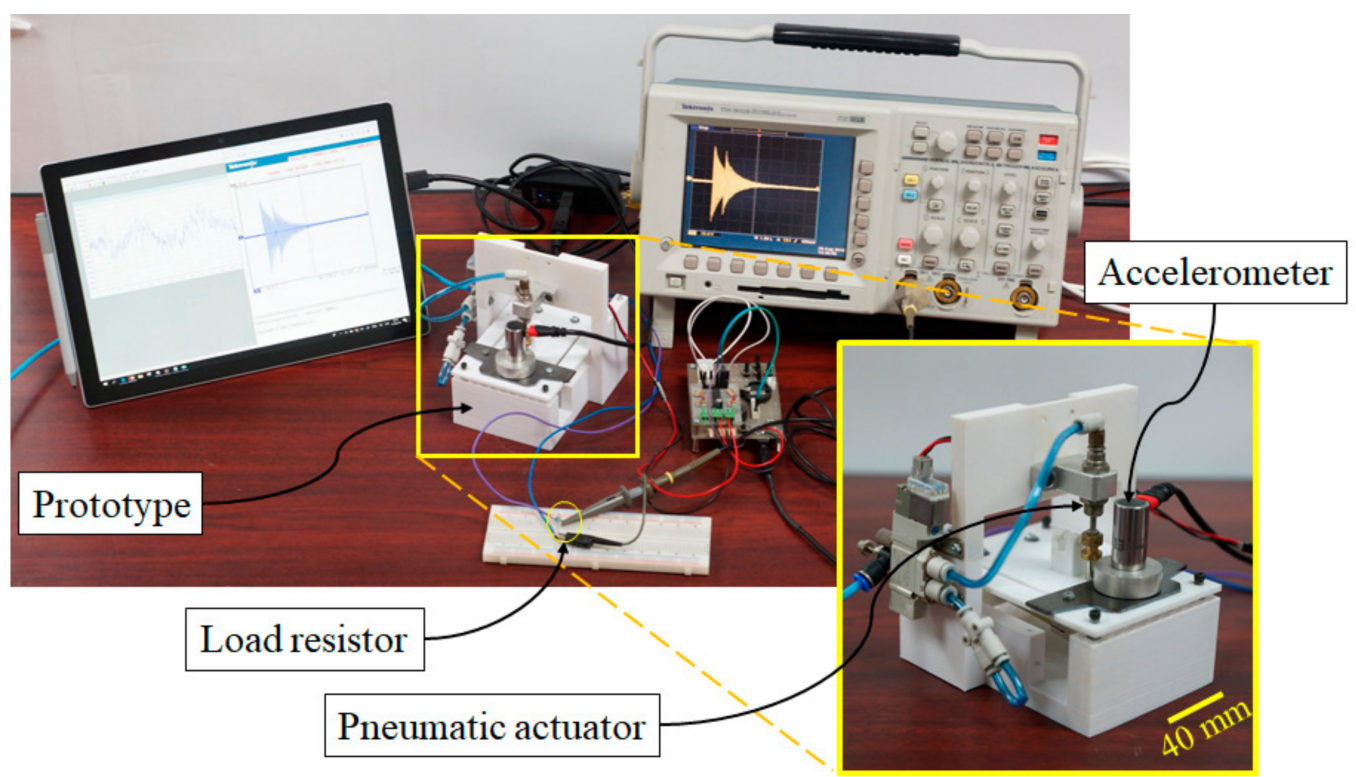

Figure 4. Test setup showing a measurement of output voltage and acceleration.

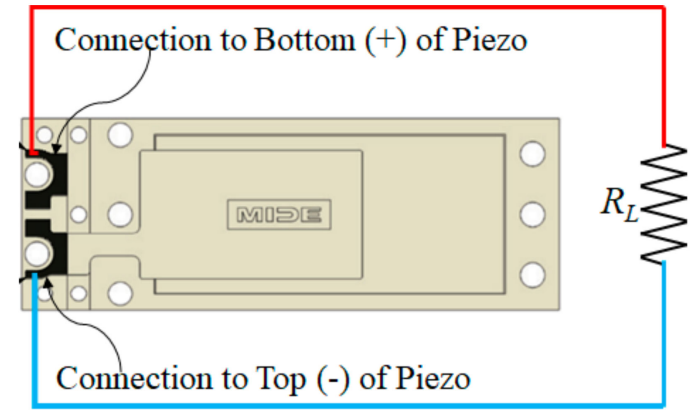

Figure 5. The piezoelectric bimorph cantilever with a load resistor circuit.

\section{Results and Discussion}

Figure 6 illustrates the typical waveform of the output voltage; this sample output voltage was obtained by singly exciting the prototype with an acceleration $\left(a_{\text {down }}\right)$ of $0.93 \mathrm{~g}$ and gap width $(h)$ of 
$4 \mathrm{~mm}$. As can be seen, the graph presents a sequence of voltage generation from just before the tile was stepped on, to being stepped on, to then moving up to its normal position after the foot moved up and away from the tile. In the first stage, the output voltage was zero because the cover plate was in the equilibrium state. The output voltage in the second stage was generated as the pneumatic actuator pushed the cover plate down. Then, when the compressed air in the pneumatic actuator was released, the springs at every corner of the prototype pushed the cover plate up rapidly generating an output voltage in the third stage. The peak to peak output voltages in the second and third stages were 68.2 and $66.4 \mathrm{~V}$, respectively. The oscillation period $(T)$ of the piezoelectric cantilever was approximately $0.071 \mathrm{~s}$, and was calculated from the distance between the output voltage peaks observed in the oscilloscope; the resonant frequency $\left(f_{r}\right)$ of the piezoelectric cantilever was therefore $14.08 \mathrm{~Hz}$ since $f_{r}=1 / T$ [30]. Throughout the experiment, the prototype was monitored and verified that it was truly able to harvest energy in double-stage frequency up-conversion with a single footstep, as designed and mentioned in Section 1.

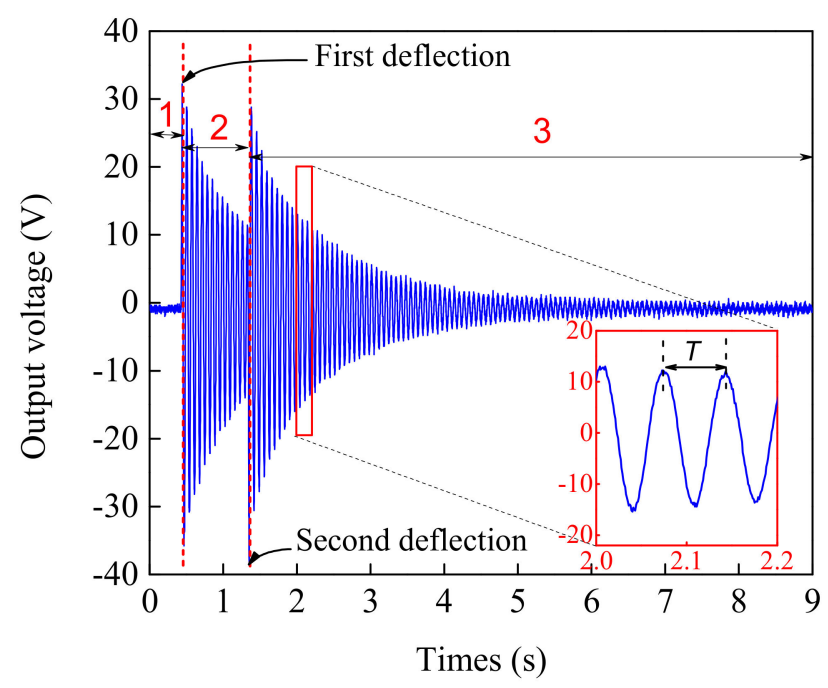

Figure 6. Typical output voltage waveform.

The results of the first experiment are illustrated in Figure 7. By pushing the cover plate of the prototype down with three acceleration values $\left(a_{\text {down }}\right)$ of $0.50,0.71$, and $0.93 \mathrm{~g}$ (arbitrarily chosen in the range of normal walking acceleration), to impact the stopper at $4 \mathrm{~mm}$ from its equilibrium position before it was released, the average output powers and energies were found to be $0.47,0.72$, and 0.82 $\mathrm{mW}$ and $1.41,2.20$, and $2.40 \mathrm{~mJ}$, respectively, at an optimal load resistance of approximately $55.68 \mathrm{k} \Omega$. The energy derived from the first-stage excitation increased as acceleration $\left(a_{\text {down }}\right)$ increased, as shown in Figure $7 \mathrm{~b}$. The amount of energy built up from the first stage increased for a very short period in the second stage and then plateaued out until the end. This might be because the springs pushed the cover plate up with nearly identical force every time they restored themselves. To conclude, increasing the acceleration of the cover plate (i.e., representing a heavier pedestrian stepping on the tile) did lead to an increase in output power and energy. The voltage and output power were plotted versus the impact force, as shown in Figure 8. The results showed a nonlinear behavior due to the large deformation on the piezoelectric cantilever beam.

In the second experiment, the energy harvesting performance of the prototype was investigated with increasing gap widths between the cover plate and stoppers: $h=2,3$, and $4 \mathrm{~mm}$. The cover plate moved down with a fixed acceleration $\left(a_{\text {down }}\right)$ of $0.93 \mathrm{~g}$ and then released. The results are shown in Figure 9. The average output power and energy observed at the optimal load resistance of $55.68 \mathrm{k} \Omega$ increased from 0.30 to 0.49 to $0.82 \mathrm{~mW}$ and 0.96 to 1.67 to $2.40 \mathrm{~mJ}$, respectively. Shown in Figure $9 \mathrm{~b}$, the steady increase in energy generated during the first and second-stage excitation was caused by an increased gap width $h$, which led to a higher impact force between the cover plate and stoppers and 
a higher restoring force of the springs pushing the cover plate up. However, too large a gap width $h$ can make walking on the tiles too uncomfortable. In practical use, the gap width should be optimized between high energy generation and comfortable walking.
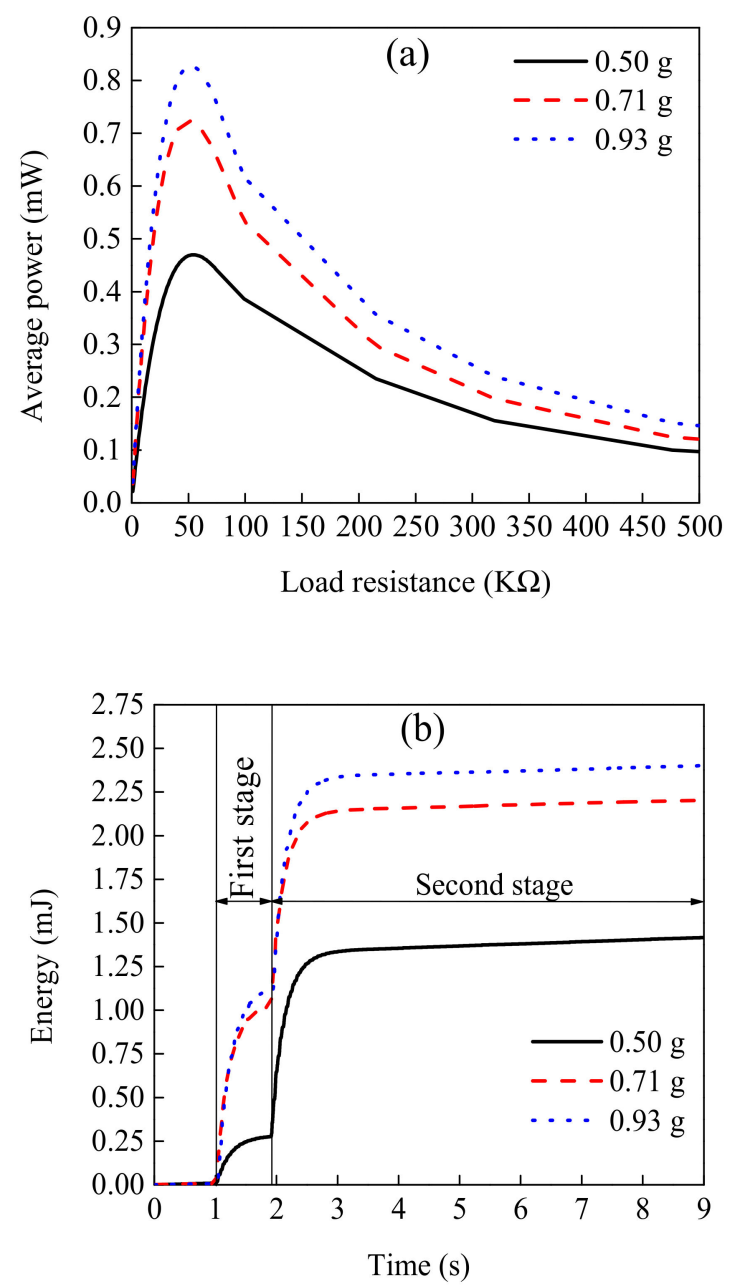

Figure 7. (a) Average output power as a function of load resistance and (b) energy at optimal load resistor generated with different acceleration $\left(a_{\text {down }}\right)$ values for a fixed displacement $(h)$.

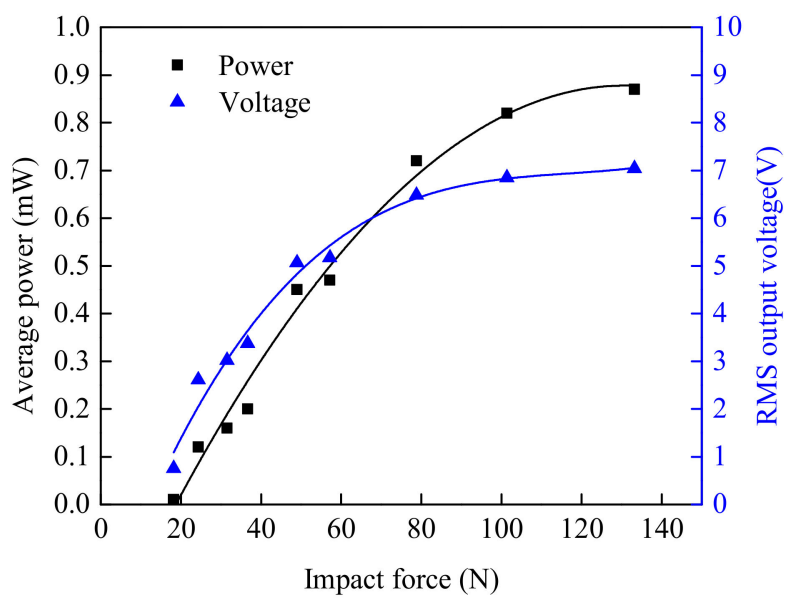

Figure 8. Average output power and RMS output voltage versus the impact force between a cover plate and the stoppers. 

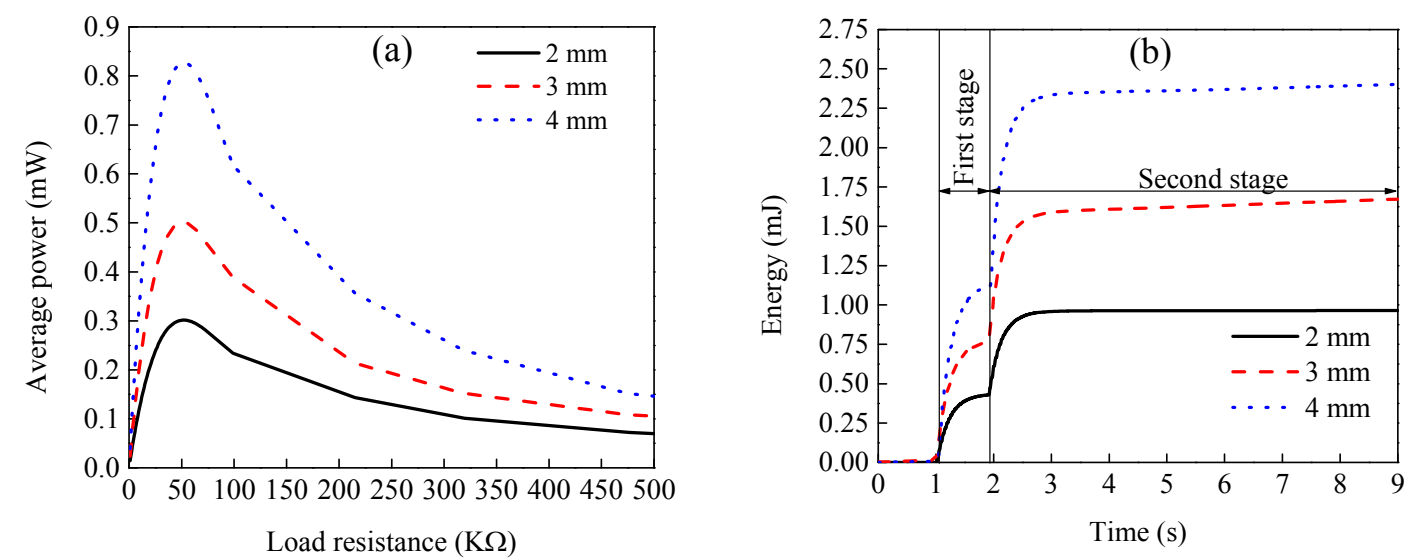

Figure 9. (a) Average output power as a function of load resistance and (b) energy at optimal load resistor generated with a fixed level of acceleration $\left(a_{\text {down }}\right)$ for several displacement distances $(h)$.

For the final experiment, the gap width $h$ was set as $4 \mathrm{~mm}$, and the cover plate was excited with an acceleration $\left(a_{\text {down }}\right)$ of $0.93 \mathrm{~g}$ at the frequencies ranging from 0.50 to $10 \mathrm{~Hz}$. Each excitation mode was in operation for $10 \mathrm{~s}$, and the energies generated at the load resistance of $55.68 \mathrm{k} \Omega$ are shown in Figure 10a-h. The highest output energy of $19.44 \mathrm{~mJ}$ was achieved at the input excitation frequency of $0.75 \mathrm{~Hz}$, not at the highest frequency tested. A drop in the generated energies with the input excitation frequencies between $1-10 \mathrm{~Hz}$ might be due to the oscillating piezoelectric cantilever resisting the input excitation force, which can be observed from the decrease in the peak generated voltage when the moving cover plate impacted the stoppers, as depicted in Figure 10c-h. Finally, the cover plate was excited with a frequency of $14 \mathrm{~Hz}$, very close to the resonant frequency of the piezoelectric cantilever, and the acceleration $\left(a_{\text {down }}\right)$ was reduced to $0.50 \mathrm{~g}$ to prevent the cover plate from hitting the oscillating piezoelectric cantilever too hard. The results showed that the output voltage almost became a pure sine wave with a peak-to-peak value of $119.2 \mathrm{~V}$, and the output energy significantly increased up to $257.57 \mathrm{~mJ}$ within approximately $10 \mathrm{~s}$, as illustrated in Figure 10i. As the input excitation frequencies increase, the harvester will exhibit a similar response as the resonant-type generator. Throughout all of these experiments, the double-stage energy harvesting floor tile demonstrated that it was able to provide a good energy harvesting performance at a low frequency and even better near the resonant frequency of the cantilever. Although, in a real scenario, the frequency of people stepping on a tile would likely be no higher than $2.5 \mathrm{~Hz}$ [31].
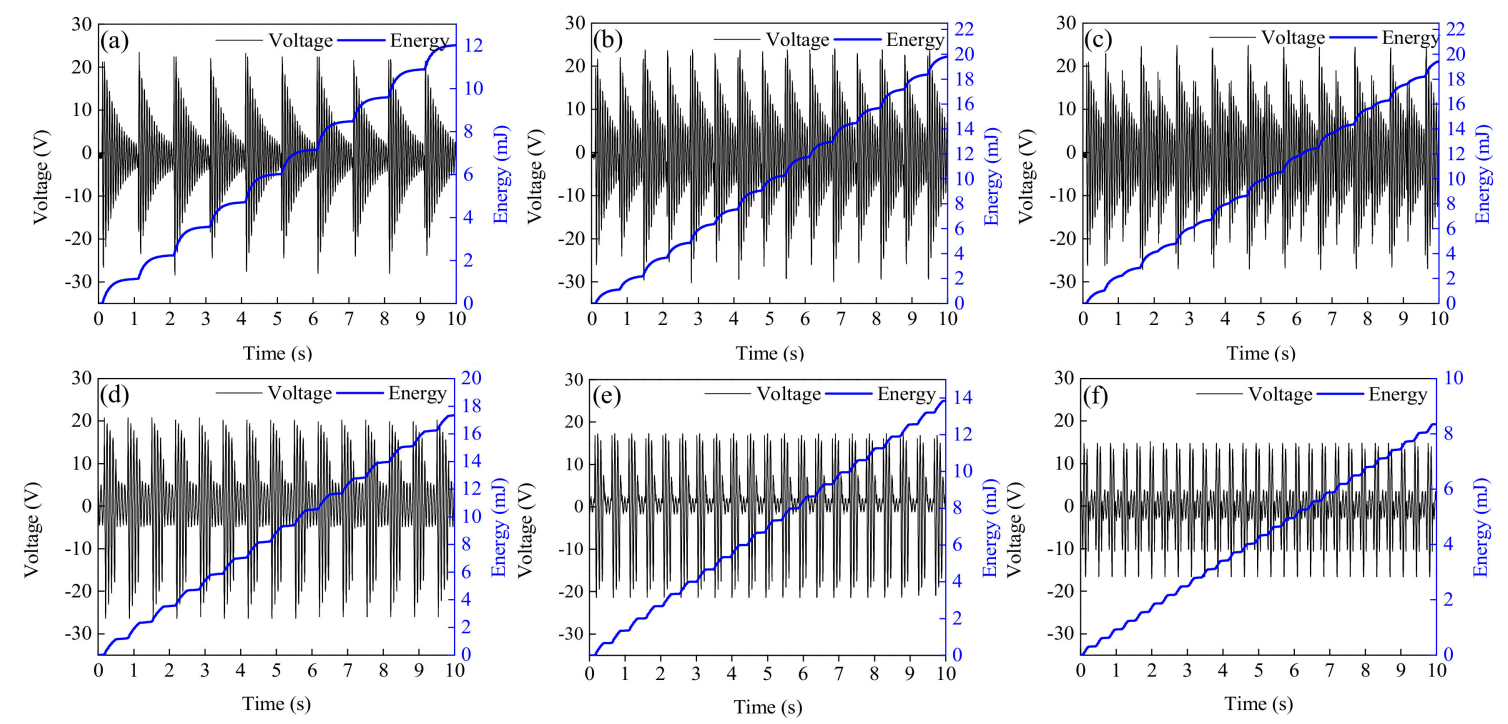

Figure 10. Cont. 

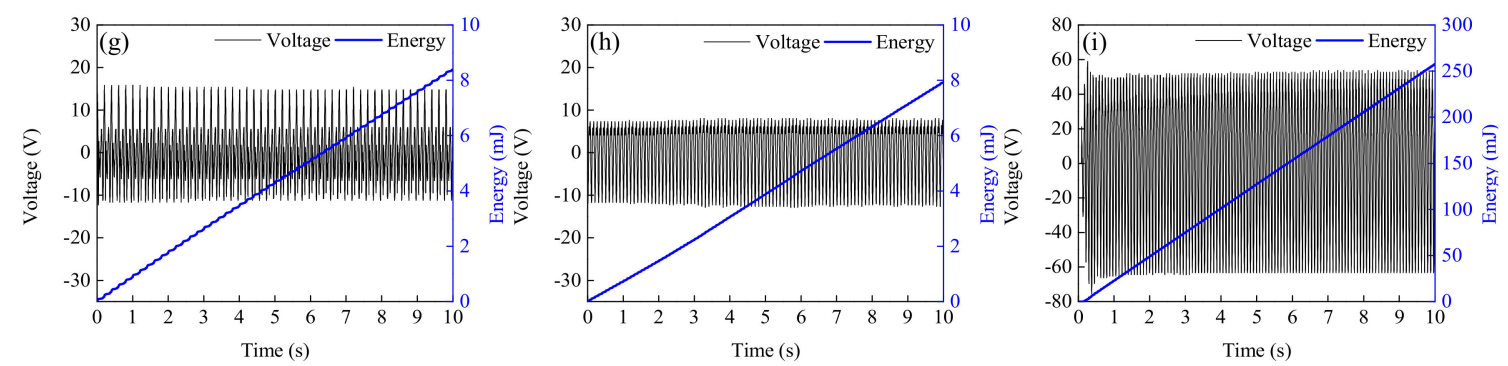

Figure 10. Voltages and energies generated with various input excitation frequencies (a) 0.5 , (b) 0.75 , (c) 1, (d) 1.5, (e) 2, (f) 2.5, (g) 5, (h) 10, and (i) $14 \mathrm{~Hz}$.

\section{Conclusions and Future Work}

The design and evaluation of a double-stage energy harvesting floor tile were proposed in this paper. The use of the mechanical impact between a cover plate and stoppers and the restoring force of springs to push a proof mass to overshoot its equilibrium position were able to achieve a frequency up-conversion. The overshooting proof mass initially made the piezoelectric cantilever deflect and was then left to freely oscillate at its resonant frequency. In the evaluation of the feasibility of the designed mechanism, one piezoelectric cantilever was prototyped and investigated with three kinds of simulated footsteps. The results show that the fabricated prototype was able to produce energy in two stages with a single landing footstep, i.e., as a foot landed on the floor tile and moved up and away from it. The output power and energy increased with an increase in the downward acceleration of the cover plate and with an increase in the gap width between the cover plate and stoppers. Moreover, the tile exhibited a good energy harvesting performance over a wide range of frequencies.

In a future study, a scaled-up version of the double-stage energy harvesting floor tile with several piezoelectric cantilevers would be tested in a real-world environment for powering low-power electronic devices for a smart city, e.g., a wireless accelerometer sensor monitoring structural conditions, advertising electronic paper display, or a wireless device monitoring pedestrian flow. Several parameters including the gap width between the cover plate and the stoppers, the size of the spring, the dimensions of the floor tile, and the weight of the proof mass would be optimized to achieve a higher energy conversion efficiency.

Author Contributions: Conceptualization, D.I.; data curation, S.J.; supervision, K.H.; validation, P.J.; writing_-original draft, P.P.

Funding: This work was supported by the Faculty of Engineering, King Mongkut's Institute of Technology Ladkrabang under the contract number 2562-02-01-022.

Conflicts of Interest: The authors declare no conflict of interest.

\section{References}

1. Miles, R.W.; Hynes, K.M.; Forbes, I. Photovoltaic Solar Cells: An Overview of State-of-the-Art Cell Development and Environmental Issues. Prog. Cryst. Growth Charact. Mater. 2005, 51, 1-42. [CrossRef]

2. Vullers, R.J.M.; van Schaijk, R.; Doms, I.; Hoof, C.V.; Mertens, B. Micropower energy harvesting. Solid State Electron. 2009, 53, 684-693. [CrossRef]

3. Beeby, S.P.; Tudor, M.J.; White, N.M.; Beeby, S. Energy harvesting vibration sources for microsystems applications. Meas. Sci. Technol. 2006, 17, R175-R195. [CrossRef]

4. Glynne-Jones, P.; Tudor, M.; Beeby, S.; White, N.; Beeby, S. An electromagnetic, vibration-powered generator for intelligent sensor systems. Sens. Actuators A Phys. 2004, 110, 344-349. [CrossRef]

5. Mitcheson, P.; Miao, P.; Stark, B.; Yeatman, E.; Holmes, A.; Green, T. MEMS electrostatic micropower generator for low frequency operation. Sens. Actuators A Phys. 2004, 115, 523-529. [CrossRef]

6. Isarakorn, D.; Briand, D.; Janphuang, P.; Sambri, A.; Gariglio, S.; Triscone, J.-M.; Guy, F.; Reiner, J.W.; Ahn, C.H.; De Rooij, N.F. The realization and performance of vibration energy harvesting MEMS devices based on an epitaxial piezoelectric thin film. Smart Mater. Struct. 2011, 20, 025015. [CrossRef] 
7. Sudhawiyangkul, T.; Isarakorn, D. Design and realization of an energy autonomous wireless sensor system for ball screw fault diagnosis. Sens. Actuat. A Phys. 2017, 258, 49-58. [CrossRef]

8. Gou, W.; Fan, S.; Geng, L. Three-layer Structure Triboelectric Electricity Generator with ULP Rectifier for Mechanical Energy Harvester. In 2018 1st Workshop on Wide Bandgap Power Devices and Applications in Asia (WiPDA Asia); Institute of Electrical and Electronics Engineers (IEEE): Xi'an, China, 2018; pp. 15-17.

9. Bertacchini, A.; Larcher, L.; Lasagni, M.; Pavan, P. Ultra low cost triboelectric energy harvesting solutions for embedded sensor systems. In Proceedings of the 2015 IEEE 15th International Conference on Nanotechnology (IEEE-NANO); Institute of Electrical and Electronics Engineers (IEEE), Rome, Italy, 27-30 July, 2015; pp. 1151-1154.

10. Azad, P. Triboelectric nanogenerator based on vertical contact separation mode for energy harvesting. In Proceedings of the 2017 International Conference on Computing, Communication and Automation (ICCCA); Institute of Electrical and Electronics Engineers (IEEE), Greater Noida, India, 5-6 May 2017; pp. 1499-1502.

11. Bertacchini, A.; Lasagni, M.; Sereni, G.; Larcher, L.; Pavan, P. Characterization and Modeling of Low-Cost Contact-Mode Triboelectric Devices for Energy Harvesting. In Proceedings of the IECON 2018 —44th Annual Conference of the IEEE Industrial Electronics Society; Institute of Electrical and Electronics Engineers (IEEE), Washington, DC, USA, 21-23 October 2018; pp. 4213-4218.

12. Kim, S.; Towfeeq, I.; Dong, Y.; Gorman, S.; Rao, A.M.; Koley, G. P(VDF-TrFE) Film on PDMS Substrate for Energy Harvesting Applications. Appl. Sci. 2018, 8, 213. [CrossRef]

13. Dorsch, P.; Bartsch, T.; Hubert, F.; Milosiu, H.; Rupitsch, S.J. Implementation and Validation of a Two-Stage Energy Extraction Circuit for a Self Sustained Asset-Tracking System. Sensors 2019, 19, 1330. [CrossRef]

14. Roundy, S.; Wright, P.K. A piezoelectric vibration based generator for wireless electronics. Smart Mater. Struct. 2004, 13, 1131-1142. [CrossRef]

15. Roundy, S.; Wright, P.K.; Rabaey, J. A study of low level vibrations as a power source for wireless sensor nodes. Comput. Commun. 2003, 26, 1131-1144. [CrossRef]

16. Gao, X.; Shih, W.-H.; Shih, W.Y. Induced voltage of piezoelectric unimorph cantilevers of different nonpiezoelectric/piezoelectric length ratios. Smart Mater. Struct. 2009, 18, 125018. [CrossRef]

17. Gu, L. Low-frequency piezoelectric energy harvesting prototype suitable for the MEMS implementation. Microelectron. J. 2011, 42, 277-282. [CrossRef]

18. Galchev, T.; Aktakka, E.E.; Najafi, K. A Piezoelectric Parametric Frequency Increased Generator for Harvesting Low-Frequency Vibrations. J. Microelectromech. Syst. 2012, 21, 1311-1320. [CrossRef]

19. Luong, H.T.; Goo, N.S. Use of a magnetic force exciter to vibrate a piezocomposite generating element in a small-scale windmill. Smart Mater. Struct. 2012, 21, 25017. [CrossRef]

20. Pillatsch, P.; Yeatman, E.; Holmes, A.S. A piezoelectric frequency up-converting energy harvester with rotating proof mass for human body applications. Sens. Actuators A Phys. 2014, 206, 178-185. [CrossRef]

21. Alghisi, D.; Dalola, S.; Ferrari, M.; Ferrari, V. Triaxial ball-impact piezoelectric converter for autonomous sensors exploiting energy harvesting from vibrations and human motion. Sens. Actuators A Phys. 2015, 233, 569-581. [CrossRef]

22. Halim, M.A.; Park, J.Y. Theoretical modeling and analysis of mechanical impact driven and frequency up-converted piezoelectric energy harvester for low-frequency and wide-bandwidth operation. Sens. Actuators A Phys. 2014, 208, 56-65. [CrossRef]

23. Chen, S.; Ma, L.; Chen, T.; Liu, H.; Sun, L. A magnetic force induced frequency-up-conversion energy harvesting system. In Proceedings of the 2016 12th IEEE/ASME International Conference on Mechatronic and Embedded Systems and Applications (MESA), Auckland, New Zealand, 29-31 August 2016; pp. 1-5.

24. Fu, H.; Khodaei, Z.S.; Aliabadi, M.F. Broadband Energy Harvesting Using Bi-Stability and Frequency Up-Conversion for Self-Powered Sensing in Internet of Things. In Proceedings of the 2019 20th International Conference on Solid-State Sensors, Actuators and Microsystems \& Eurosensors XXXIII (TRANSDUCERS \& EUROSENSORS XXXIII); Institute of Electrical and Electronics Engineers (IEEE), Berlin, Germany, 23-27 June 2019; pp. 354-357.

25. Panthongsy, P.; Isarakorn, D.; Janphuang, P.; Hamamoto, K. Fabrication and evaluation of energy harvesting floor using piezoelectric frequency up-converting mechanism. Sens. Actuators A Phys. 2018, 279, 321-330. [CrossRef] 
26. Pozzi, M.; Zhu, M. Characterization of a rotary piezoelectric energy harvester based on plucking excitation for knee-joint wearable applications. Smart Mater. Struct. 2012, 21, 55004. [CrossRef]

27. Janphuang, P.; Lockhart, R.A.; Isarakorn, D.; Henein, S.; Briand, D.; de Rooij, N.F. Harvesting Energy From a Rotating Gear Using an AFM-Like MEMS Piezoelectric Frequency Up-Converting Energy Harvester. J. Microelectromech. Syst. 2015, 24, 742-754. [CrossRef]

28. Kuang, Y.; Yang, Z.; Zhu, M. Design and characterisation of a piezoelectric knee-joint energy harvester with frequency up-conversion through magnetic plucking. Smart Mater. Struct. 2016, 25, 85029. [CrossRef]

29. Mide Technology. PPA Products Datasheet-User Manual. Available online: https://info.mide.com/piezoproducts/download-piezo-products-datasheets (accessed on 2 June 2019).

30. Panthongsy, P.; Isarakorn, D.; Hamamoto, K. A Test Bench for Characterization of Piezoelectric Frequency Up-Converting Energy Harvesters. In Proceedings of the 2018 15th International Conference on Electrical Engineering/Electronics, Computer, Telecommunications and Information Technology (ECTI-CON), Chiang Rai, Thailand, 18-21 July 2018; pp. 134-137.

31. Pachi, A.; Ji, T. Frequency and velocity of people walking. Struct. Eng. 2005, 83, 36-40.

(C) 2019 by the authors. Licensee MDPI, Basel, Switzerland. This article is an open access article distributed under the terms and conditions of the Creative Commons Attribution (CC BY) license (http://creativecommons.org/licenses/by/4.0/). 